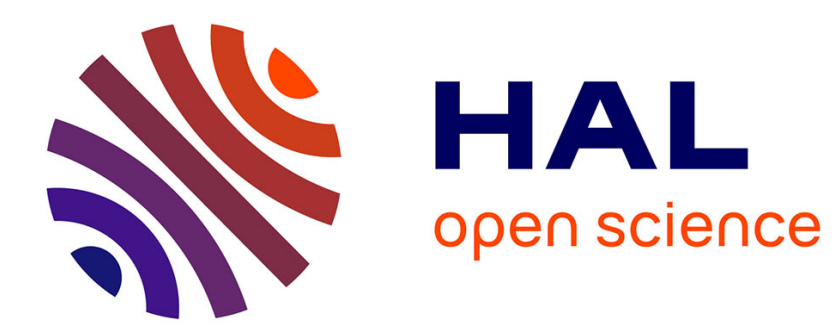

\title{
Cyclic Orders: Equivalence and Duality
}

\author{
András Sebö, Pierre Charbit
}

\section{To cite this version:}

András Sebö, Pierre Charbit. Cyclic Orders: Equivalence and Duality. 2006. hal-00005192

\section{HAL Id: hal-00005192 \\ https://hal.science/hal-00005192}

Preprint submitted on 7 Jun 2006

HAL is a multi-disciplinary open access archive for the deposit and dissemination of scientific research documents, whether they are published or not. The documents may come from teaching and research institutions in France or abroad, or from public or private research centers.
L'archive ouverte pluridisciplinaire HAL, est destinée au dépôt et à la diffusion de documents scientifiques de niveau recherche, publiés ou non, émanant des établissements d'enseignement et de recherche français ou étrangers, des laboratoires publics ou privés. 


\section{Les cahiers Leibniz}

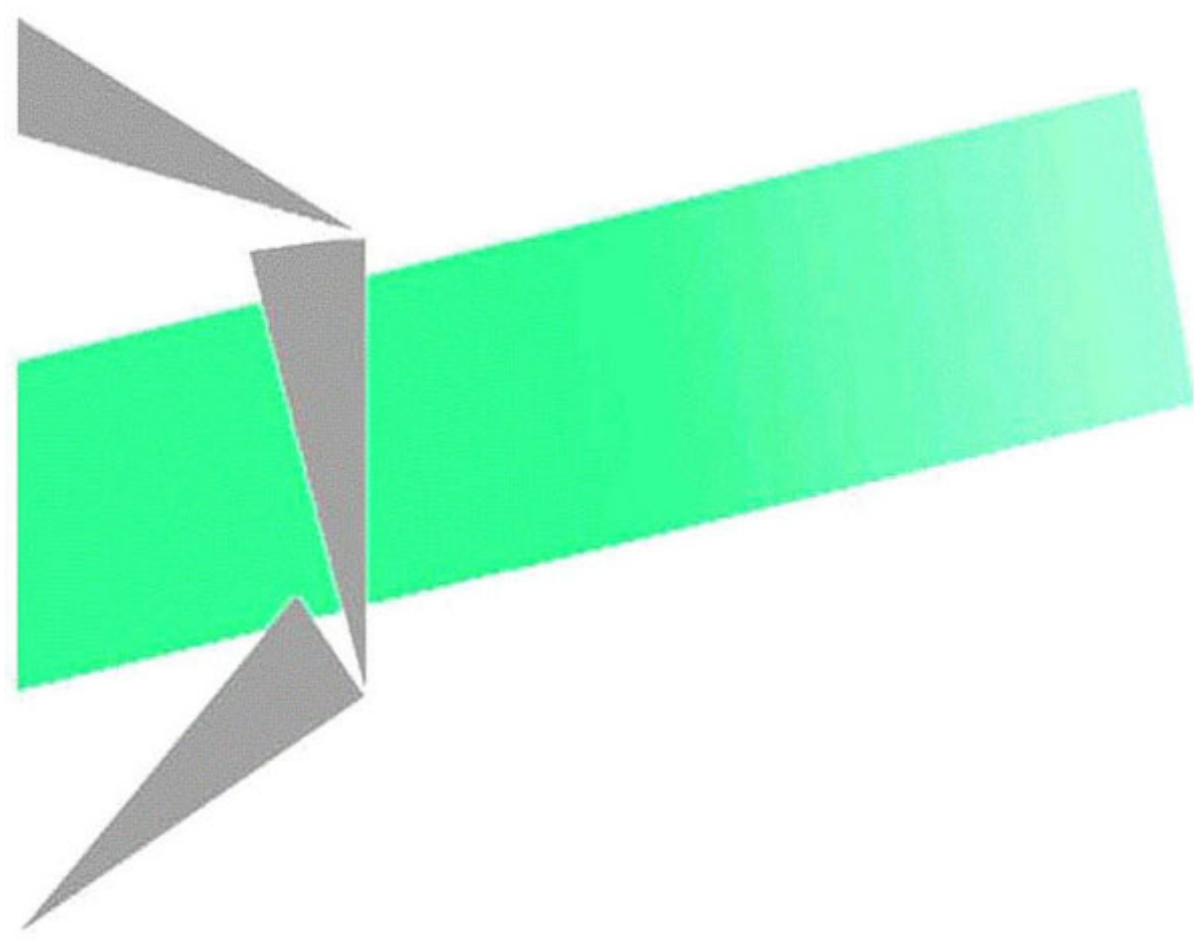

Cyclic Orders: Equivalence and Duality

Pierre Charbit and András Sebö

Laboratoire Leibniz-IMAG, 46 av. Félix Viallet, 38000 GRENOBLE, France ISSN : 1298-020X $n^{\circ} 119$

Avr 2005

Site internet : http://www-leibniz.imag.fr 



\title{
Cyclic Orders: Equivalence and Duality
}

\author{
Pierre Charbit* András Sebő†
}

March 24, 2005

\begin{abstract}
Cyclic orders of graphs and their equivalence have been promoted by Bessy and Thomassés recent proof of Gallai's conjecture. We explore this notion further : we prove that two cyclic orders are equivalent if and only if the winding number of every circuit is the same in the two. The proof is short and provides a good characterization and a polynomial algorithm for deciding whether two orders are equivalent.

We then derive short proofs of Gallai's conjecture and a "polar" result of Bessy and Thomassé's using the duality theorem of linear programming, total unimodularity, and the new result on the equivalence of cyclic orders.
\end{abstract}

\section{Introduction}

In this paper we characterize - with a simple good-characterization and polynomial algorithm the "equivalence" of (linear or cyclic) orders given on the vertices of a directed graph. This notion has been introduced by Bessy and Thomassé [1] in order to prove a forty years old conjecture of Gallai, and seems to be a basic concept that can be expected to have further applications.

Furthermore, we show the linear programming background of Bessy and Thomassé's results. They prove two minmax theorems that are in 'antiblocking relation', and the related polyhedra have advantageous integrality properties that can be handled algorithmically with network flows [5]. We provide here proofs that are the simplest to our knowledge: these are based on total unimodularity and linear programming duality without any concern of how the solutions of these are found. The proof of the second theorem uses the characterization of equivalent orders.

If $D$ is a digraph then the underlying graph is the undirected graph $G=G(D)$ whose edges are the $\operatorname{arcs}$ of $D$ without orientation. We will say that $D$ is connected or 2-edge-connected, if the underlying graph has these properties. (Note the difference with strongly connected digraphs, which is a property of the digraph. For the standard definitions of graph theory or polyhedral combinatorics we refer to [4].) Strongly connected digraphs will shortly be said to be strong.

A cycle is a closed walk with distinct arcs (edges) and it is a circuit if all vertices are distinct, both in directed and undirected graphs. We will use the term undirected circuits of a digraph to design the circuits of the underlying graph. (The orientation of the arcs of such a circuit can be arbitrary.) A multiset is a set where each element has a nonnegative integer multiplicity. A linear order of a (di)graph is an order $O:=\left(v_{1}, v_{2}, \ldots, v_{n}\right)$ of its vertices. If in addition $v_{n}$ is followed by $v_{1}$, we call it a cyclic order. A cyclic order of $n$ elements, has $n$ openings $\left(v_{i}, v_{i+1}\right)(i=1, \ldots, n)$, that is, linear orders (starting with $v_{i+1}$ and ending with $v_{i}$ ) which are cyclically equal to it. For a

\footnotetext{
${ }^{*}$ Université Claude Bernard, Lyon

${ }^{\dagger}$ CNRS, Leibniz-IMAG, Grenoble
} 
notation, a cyclic order can be represented by any of its openings. A cyclic shift of a linear order is another opening of the same cyclic order.

All digraphs considered here are without loops or parallel arcs, but may have directed 2-circuits, where a $k$-circuit $(k \in \mathbb{N})$ is a circuit of size $k$. A digraph is a pair $D=(V, A)$, where $V=V(D)$ is the vertex-set of $D$, and $A=A(D)$ is its arc-set. An arc $a=u v \in A$ has a head $h(a):=v$ and a tail $t(a):=u$. Sets $X \subseteq S$ will also denote their $0-1$ incidence (characteristic) vectors in $\{0,1\}^{S}$.

Given a digraph $D=(V, A)$, a circulation is a function $f: A \longrightarrow \mathbb{R}$ such that $f\left(\delta^{\text {in }}(x)\right)=$ $f\left(\delta^{\text {out }}(x)\right)$ for all $x \in V$, where $\delta^{\text {in }}(x)$ and $\delta^{\text {out }}(x)$ is the set of arcs entering, respectively leaving $x$. We do not require $f$ to have nonnegative values. Clearly, any of the two \pm 1 vectors associated to any undirected circuit of $D$ (putting 1 on arcs in one direction, and -1 on those in the opposite direction) and any of their linear combinations are circulations; it is well-known and easy to see that conversely, any circulation is the linear combination of circuits signed in this way.

Given a cyclic order $\left(v_{1}, v_{2}, \ldots, v_{n}\right)$, the length of an $\operatorname{arc}\left(v_{i}, v_{j}\right)$ is $j-i$ if $j>i$ and $n+j-i$ if $i>j$. If $C$ is a cycle of $D$, the sum of the lengths of its $\operatorname{arcs}$ is a multiple $\operatorname{ind}(C) n$ of $n$. This integer ind $(C)$ is called the index (winding number) of $C$. The index $\operatorname{ind}(\mathcal{C})$ of a family $\mathcal{C}$ of cycles is the sum of the indices of its constituent cycles. A circuit of index 1 is called a round. If every arc lies in a round, the cyclic order is said to be coherent. Bessy and Thomassé [1] showed that every strong digraph has a coherent cyclic order.

In the linear order $\left(v_{1}, v_{2}, \ldots, v_{n}\right)$ an arc $v_{i} v_{j}$ is called a forward arc if $i<j$, and backward arc if $i>j$. It is an important observation in [1] that the index of a circuit is equal to the number of its backward arcs in any opening; in particular, the number of backward arcs of a circuit does not change through cyclic shifts.

If $G$ is an undirected graph with a cyclic order, we can also define the index for its undirected circuits : the vertices of every undirected circuit can be ordered in two ways (so that consecutive vertices correspond to edges of $C$ ). The index $\operatorname{ind}(C)$ of $C$ with either of these orders is the index of the corresponding directed circuit; equivalently it is equal to the number of backward arcs in any opening of the cyclic order. Note that the sum of the indices of the two orders of $C$ (with respect to the fixed cyclic order of $G$ ) is $|C|$, and therefore one of these determines the other.

\section{Equivalence of Cyclic Orders}

We first introduce the fundamental equivalence classes of cyclic orders promoted by Bessy and Thomassé's proofs [1]. Then we establish a basic invariance property of these equivalence classes, that will be used later on.

Suppose $G=(V, E)$ is an undirected graph. We will also use the following notions for directed graphs, but ignoring the orientation of the arcs. Two cyclic orders are equivalent if one can be obtained from the other by a sequence of elementary operations. An elementary operation is a permutation (interchange) of nonadjacent consecutive vertices, that is $\left(v_{1}, v_{2}, \ldots, v_{n}\right)$ maps to $\left(v_{2}, v_{1}, \ldots, v_{n}\right)$, where $v_{1}$ and $v_{2}$ are nonadjacent.

The motivation for this definition is that it preserves the index of every circuit of $G$. We show in this paper that two cyclic orders of a strongly connected digraph are equivalent if and only if all circuits have the same index in the two.

This does not hold for arbitrary digraphs: an acyclic orientation of a triangle has two nonequivalent cyclic orders, even though it does not even have directed cycles. We first prove a stronger condition involving all undirected circuits to be necessary and sufficient for arbitrary digraphs. 
Theorem 2.1 Let $G=(V, E)$ be an undirected graph. Let $O_{1}$ and $O_{2}$ be two cyclic orders of $V$. The following statements are equivalent:

(i) $\mathrm{O}_{1} \sim \mathrm{O}_{2}$.

(ii) The index of every circuit is the same with respect to $\mathrm{O}_{1}$ and $\mathrm{O}_{2}$.

It follows for instance that for forests any two cyclic orders are equivalent (which is easy to check directly). Note that this theorem provides a good characterization (a linear $N P \cap \operatorname{coNP}$ certificate) for two orders to be equivalent. It is not surprising that this certificate depends only on the underlying undirected graph: the elementary operations depend only on this graph.

Note that this theorem has no condition on $G$. For strongly connected graphs the condition on directed cycles will turn out to imply the condition for undirected cycles.

Proof : Since an elementary operation does clearly not change the index of a circuit or of a closed walk, (i) implies (ii).

Let us prove now the essential statement "(ii) implies (i)", by induction on the number of edges. Let $e=x y \in E(x, y \in V)$ be an arbitrary edge.

By the induction hypothesis the statement is true for $G-e$, that is, there exists a sequence $\pi_{1}, \ldots, \pi_{k}$ of elementary operations that brings the order $O_{1}$ to the order $O_{2}$ in $G-e$. Every elementary operation on $G-e$ is also an elementary operation of $G$, except the permutation of $x$ and $y$. If this operation does not occur, we are done: we have a sequence of elementary operations that brings $O_{1}$ to $O_{2}$.

Claim: If the permutation of $x$ and $y$ does occur among $\pi_{1}, \ldots, \pi_{k}$, then there exist $O_{1}^{\prime}, O_{2}^{\prime}$, $O_{1}^{\prime} \sim O_{1}, O_{2}^{\prime} \sim O_{2}$ such that $x$ is followed by $y$ in both $O_{1}^{\prime}$ and $O_{2}^{\prime}$, or $y$ is followed by $x$ in both.

This Claim finishes the proof of the theorem: since $e$ joins neighboring vertices in both $O_{1}^{\prime}$ and $O_{2}^{\prime}$, and in the same order, these orders obviously define orders $O_{1}^{\prime \prime}, O_{2}^{\prime \prime}$ of $G / e$ (the graph obtained after contraction of $e$ ); furthermore, since (i) implies (ii) (and this is already proven), the condition (ii) is still satisfied for $O_{1}^{\prime}$ and $O_{2}^{\prime}$, and therefore for $O_{1}^{\prime \prime}$ and $O_{2}^{\prime \prime}$ as well. Since $G / e$ has less edges than $G$, by the induction hypothesis $O_{1}^{\prime \prime} \sim O_{2}^{\prime \prime}$, and the elementary operations of $G / e$ correspond obviously to one or two elementary operations of $G$.

In order to prove the Claim let $i, j, 1 \leq i \leq j \leq k$ be the first and the last index where the permutation of $x$ and $y$ occurs. Let $O_{1}^{\prime}$ be the cyclic order we get from $O_{1}$ if we stop before executing $\pi_{i}$, and $O_{2}^{\prime}$ the order we get by executing the permutations in reverse order from $O_{2}$ and stopping before executing $\pi_{j}$. Clearly, $O_{1}^{\prime} \sim O_{1}, O_{2}^{\prime} \sim O_{2}$, and therefore $O_{1}^{\prime}, O_{2}^{\prime}$ satisfy (ii).

In both $O_{1}^{\prime}$ and $O_{2}^{\prime} x$ and $y$ are consecutive by definition. If they follow one another in the same order in $O_{1}^{\prime}$ and $O_{2}^{\prime}$, then we are done. If not, suppose without loss of generality (by possibly interchanging the notation $x, y)$ that $x$ precedes $y$ in $O_{1}^{\prime}$, and $y$ precedes $x$ in $O_{2}^{\prime}$.

Take a shift in $O_{2}^{\prime}$ so that $x$ is the first, and $y$ the last element. There is no forward path now from $x$ to $y$ in $G-e$, because if there was such a path $P=\left(x=x_{0}, x_{1}, \ldots, x_{p}=y\right)$, then $p \geq 2$, and with the edge $y x, P$ is in fact a cycle of index 1 . On the other hand taking an opening of $O_{1}^{\prime}$ different from $(x, y)$ we see that $y x$ is a backward arc in $P$, and there must be another backward arc since otherwise $p=1$. Therefore the index of the cycle $P$ in $O_{1}^{\prime}$ is at least 2, while it is 1 in $O_{2}^{\prime}$, contradicting (ii).

It follows that the set $X$ of vertices that can be reached from $x$ with a forward path (in $O_{2}^{\prime}$ ) have no forward neighbour outside $X$, and therefore $X$ can be placed after $y$ through a sequence of elementary changes. The vertices in $Y:=V \backslash(X \cup\{x, y\})$ have no backward neighbour in $X$, 
so similarly, they can be placed before $x$. Therefore $O_{2}^{\prime \prime}:=Y, x, y, X$ is an equivalent order, and $y$ follows $x$ as in $O_{1}$. So $O_{1}^{\prime}$ and $O_{2}^{\prime \prime}$ are as claimed.

We promised that for strongly connected digraphs condition (ii) is sufficient to hold for directed circuits in order to deduce (i). This sharpening follows by simple linear properties of circuits roughly, the circuits of a strongly connected digraph "generate" all the undirected circuits of the underlying graph:

If $D=(V, A)$ is a directed graph, then each cycle of the underlying graph can be represented as a vector in $\{-1,0,1\}^{A}$ in the following usual way (see for instance network matrices in [4]) :

Let $C$ be an undirected circuit (with one of the two orderings fixed for reference), and define the vector $\vec{C} \in\{-1,0,1\}^{A}$ as follows: $\vec{C}(a)=1$ if $a \in C$ is oriented in the sense of the orientation of $C, \vec{C}(a)=-1$ if it is oriented in the opposite sense, and $\vec{C}(a)=0$ if $a \notin C$.

$$
\mathcal{C}(D):=\operatorname{lin}\{\vec{C}: C \text { is a circuit of } G(D)\} .
$$

Note that the definition of $\mathcal{C}(D)$ does not depend on which of the two orientations of the circuits we chose, since the vector defined by the opposite orientation is just $-\vec{C} \cdot \mathcal{C}(D)$ is the set of circulations.

Lemma 2.1 A 2-edge-connected digraph $D=(V, A)$ is strongly connected if and only if $\mathcal{C}(D)$ is spanned (linearly) by the (directed) circuits of $D$ (as vectors in $\{0,1\}^{A}$ ).

Indeed, if $D$ is not strongly connected, let $e$ be a an edge not contained in a directed circuit. Then since the underlying undirected graph is 2-edge connected, there exists an undirected circuit $C$ in $G(D), e \in E(C)$; since $e$ is not contained in any directed circuit, $C$ is not generated by circuits of $D$.

Conversely suppose that $D$ is strongly connected. Then any circulation $f$ is generated by directed circuits: indeed, for each of the negative coordinates $e_{1}, \ldots, e_{p}$ of $f$ choose a circuit $C_{i}$ containing $e_{i}(i=1, \ldots, p) ; f-\sum_{i=1}^{p} f\left(e_{i}\right) C_{i}$ is a nonnegative circulation, which is obviously a (nonnegative) combination of directed circuits, and then so is $f$.

Theorem 2.2 Let $D=(V, A)$ be a strongly connected digraph. Let $O_{1}$ and $O_{2}$ be two cyclic orders of $V$. Suppose that the index of each circuit is the same with respect to $O_{1}$ and $O_{2}$. Then $O_{1} \sim O_{2}$.

Proof : We have to prove only that the condition is implied for every undirected circuit of the underlying graph, because then Theorem 2.1 implies the assertion. Denote by $\operatorname{ind}_{i}(C)$ the index of circuit $C$ according to $O_{i},(i=1,2)$.

Open both $O_{1}$ and $O_{2}$ to get the linear orders $L_{1}$ and $L_{2}$. Define the vectors $w_{1}, w_{2} \in\{1,-1\}^{A}$ to be -1 on backward arcs and 1 on forward arcs.

Note first that $w_{i}(C)=\left(|E(C)|-\operatorname{ind}_{i}(C)\right)-\operatorname{ind}_{i}(C)=|E(C)|-2 \operatorname{ind}_{i}(C)$ for every cycle $(i=1,2)$. So the assumption on the equality of indices according to the two orders is equivalent to $w_{1}(C)=w_{2}(C)$ for every circuit $C$.

The equation

$$
w_{i}^{T} \vec{C}=|E(C)|-2 \operatorname{ind}_{i}(C)
$$

holds for all the circuits of $G(D)$. Indeed, in the inner product $w_{i}^{T} \vec{C}$ we have four kinds of terms: $1 \cdot 1,1 \cdot(-1),(-1) \cdot 1,(-1) \cdot(-1)$, and it is clear that the result is 1 if the corresponding edge goes forward in $\vec{C}$, and -1 if it goes backward, and the difference of the forward and backward edges is $|E(C)|-2 \operatorname{ind}_{i}(C)$. 
So for checking that the condition (ii) of Theorem 2.1 holds, it is sufficient to prove $w_{1}^{T} \vec{C}=w_{2}^{T} \vec{C}$ for every circuit $C$ of $G(D)$. However, since we know that this holds for directed circuits, and by Lemma 2.1 the directed circuits generate $\mathcal{C}(D)$, it follows for every undirected circuit $C$ of $G(D)$, as expected.

\section{Coherent Orders and Gallai's conjecture}

\subsection{Coherent cyclic orders}

In this section we will investigate the notion of coherent cyclic orders defined in [1]. Recall that it means that each arc lies in a circuit of index 1 . We will prove, in fact, that as long as each arc lies in a circuit, the digraph admits such a cyclic order. That is why we only need to focus on strong digraphs.

We define the following reflexive and transitive relation on cyclic orders :

$$
O_{1} \leq O_{2} \text { if for each circuit } C \text { of } D, \operatorname{ind}_{O_{1}}(C) \leq i n d_{O_{2}}(C)
$$

Extend this relation to equivalence classes of cyclic orders: if $\mathcal{O}_{1}$ and $\mathcal{O}_{2}$ are two such equivalence classes, then $\mathcal{O}_{1} \leq \mathcal{O}_{2}$ if and only if there exist $O_{1} \in \mathcal{O}_{1}, O_{2} \in \mathcal{O}_{2}$ such that $O_{1} \leq O_{2}$. This relation is said to agree (with the one defined on the cyclic orders) if $\mathcal{O}_{1} \leq \mathcal{O}_{2}$ implies $O_{1} \leq O_{2}$ for all $O_{1} \in \mathcal{O}_{1}, O_{2} \in \mathcal{O}_{2}$. The following theorem is a reformulation of Theorem 2.2:

Theorem 3.1 The relation " $\leq$ " agrees, and it is a partial order.

Proof : Suppose $O_{1}, O_{2}$ are cyclic orders, and $O_{1} \leq O_{2}, O_{2} \leq O_{1}$. By definition, this means that the condition of Theorem 2.2 is satisfied, and then the theorem states that $O_{1}$ and $O_{2}$ are equivalent cyclic orders. Conversely, if $O_{1}$ and $O_{2}$ are equivalent, then $O_{1} \leq O_{2}, O_{2} \leq O_{1}$. Since the relation on cyclic orders is reflexive and transitive, it follows (easily and in a well-known way) that on the equivalence classes it agrees, and is also antisymmetric.

The following statement is equivalent to Bessy and Thomassé's key-lemma about the existence of a coherent cyclic order in strongly connected graphs [1]. The following simple proof is an adaptation of the variant in [5], nevertheless the framework of any proof can be adapted.

Proposition 3.1 Let $D$ be a strong digraph. Any order in a class that is minimal (with respect to $\leq)$ is coherent.

Indeed, let $O$ be an order and $e \in A$ an arc that is not contained in a round of $O$. By replacing $O$ with an equivalent order we can suppose that $e \in B$, where $B$ is the set of backward arcs. The set $E(G) \backslash(B \backslash e)$ does not contain a cycle, -since every cycle has a backward arc and not only $e$ -, so it has an order where every $\operatorname{arc} \operatorname{in} E(G) \backslash(B \backslash e)$ is a forward arc. In this cyclic order $O^{\prime}$ the set of backward $\operatorname{arcs} B^{\prime}$ satisfies $B^{\prime} \subseteq B \backslash e$. Clearly, for every circuit $C$ :

$$
\text { ind }^{\prime}(C)=\left|C \cap B^{\prime}\right| \leq|C \cap B|=\operatorname{ind}(C),
$$

where ind ${ }^{\prime}$ denotes the indices according to $O^{\prime}$. Since $G$ is strongly connected, $e$ is contained in a circuit $C$, and for this circuit strong inequality holds, proving that $O$ is not minimal with respect to $\leq$, and finishing the proof. 


\subsection{Index-Bounded Weightings and a Min-Max theorem}

The main result of Bessy and Thoomassé in [1] is a Conjecture of Gallai, that we will refer to as Gallai's conjecture.

Theorem 3.2 (BT - 2003) Let D be a strong digraph and denote by $\alpha$ the stability of the graph, that is the maximum cardinality of a stable set. Then the vertices of $D$ can be covered by less than $\alpha$ circuits.

This is a consequence of a minmax theorem proved by Bessy and Thomassé. In this section we provide a simple direct proof - differently from [1] and [5]- of a linear programming type corollary of Bessy and Thomassé's result which in turn easily implies Gallai's conjecture. We will use linear programming duality and total unimodularity in the proof, without any algorithmic aim.

A weighting of a digraph $D$ is a function $w: V \rightarrow \mathbb{N}$. The weight of a vertex $v$ of $D$ is the value $w(v)$. By extension, the weight of a subgraph of $D$ is the sum of the weights of its vertices. If $D$ is endowed with a cyclic ordering $O$, and if $w(C) \leq i(C)$ for every circuit $C$ of $D$, we say that the weighting $w$ is index-bounded (with respect to $O$ ). We could also say "index-bounded multiset" of vertices. We prove the following:

Theorem 3.3 Let $D$ be a digraph and suppose each of its vertices lies in a circuit, and $O$ is a cyclic order of $D$. Then

$\min \{i(\mathcal{C}): \mathcal{C}$ a circuit covering of $D\}=\max \{w(D): w$ is an index-bounded weighting $\}$.

Gallai's conjecture can be easily deduced by applying this theorem to a coherent cyclic order:

- for every family $\mathcal{C}$ of circuits of $D,|\mathcal{C}| \leq i(\mathcal{C})$;

- since each vertex is the endpoint of an arc, it is also contained in a round, and therefore an index-bounded weighting of $D$ is necessarily $(0,1)$-valued;

- There is no arc $a=v_{i} v_{j} \in A$ such that $w(i)=w(j)=1$, because the round $C$ containing $w$ satisfies $w(C) \leq 1$. So the support of $w$ is a stable set, and $w(D) \leq \alpha(D)$ follows.

Note that one gets Theorem 3.3 from Bessy and Thomassé's main minmax result ([1], Theorem 1) by replicating stable sets into vertices and relaxing coherence. It is rephrasing the "total dual integrality" of the minimal polyhedral description of index-bounded weightings [5]. Our goal here is to gain in simplicity by sacrificing the algorithm. We aim at the simplest possible proof of the same spirit as the other proofs of this paper.

Conversely, the theorem implies [1], Theorem 1 with the help of a simple combinatorial characterization of index-bounded weightings for coherent orders as "cyclic stable sets" [5].

Let us prepare the proof of Theorem 3.3 by recalling some basic well-known notations and facts:

- for a directed graph $D=(V, A), M=M(D)$ denotes the $n \times m$ incidence matrix of $D$ with entries $m_{a, v}(a \in A, v \in V)$ equal to -1 if $v$ is the tail of arc $a, 1$ if it is the head, and 0 otherwise; if the arcs or (and) the vertices of $D$ are indexed we will replace $a$ or $v$ by its index.

- The solutions $x \in \mathbb{R}^{A}$ of the equation $M x=0$ are circulations.

- Given a function $d: A \longrightarrow \mathbb{R}$ the solutions of $\pi M \leq d, \pi \in \mathbb{R}^{V}$ are called potentials (for $d$ ). 
- Given $d$, there exists no negative cycle according to $d$ if and only if $d^{\top} x \geq 0$ for every circulation, and this holds if and only if (by Farkas's Lemma) there exists a potential for $d$. (Here we need only these facts, and can ignore the well-known combinatorial procedures that compute circulations or potentials [4].)

These are conform to notations and terminology in [4] which is a reference for more details or proofs, if necessary. Denote $M^{+}=M^{+}(D)$ the $n \times m$ matrix with entries $m_{a, v}^{+}=\max \left\{m_{a, v}, 0\right\}$ $(a \in A, v \in V)$.

It is easy to see that the $2 n \times m$ matrix $\widetilde{M}$ whose first $n$ rows constitute a matrix identical to $M$ and the second $n$ rows a matrix identical to $M^{+}$is totally unimodular: indeed, in any square submatrix $M^{\prime}$, subtract the $i$-th row of $M^{+}$from the $i$-th row of $M$ for all $i$ for which both rows are present in $M^{\prime}$. We get a matrix with at most two nonzeros per column, and if there are two nonzeros, then one of them is 1 the other -1 ; such a matrix is the submatrix of the incidence matrix of a graph, and as such, has determinant 0 or \pm 1 , and the determinant of $M^{\prime}$ is the same.

\subsection{Proof of Theorem 3.3}

Let $D$ be a digraph, with vertex set $V$ and an opening $\left(v_{1}, \ldots, v_{n}\right)$ of the cyclic order $O$. We note its arc set $A=\left\{a_{1}, \ldots, a_{m}\right\}$, and define the objective function $c \in \mathbb{R}^{m}$ with $c_{i}:=1$ if $a_{i}$ is a backward arc and 0 otherwise. Consider the linear program $x \in \mathbb{R}^{m}$,

$$
\text { minimize } c^{\top} x \text { subject to } M x \geq 0, M^{+} x \geq 1, x \geq 0 \quad(P) \text {. }
$$

Since $\widetilde{M}$ is totally unimodular, the linear program $(\mathrm{P})$ has integer primal and dual optima and by the duality theorem of linear programming [4] the two optima are equal.

Claim 1: The primal optimum of $(\mathrm{P})$ is equal to the left hand side of the minmax equality.

Let $x$ be an integer primal solution (with objective value $c^{\top} x$ ). We first show that $x$ is an integer circulation: indeed, the sum of the rows of $M$ is 0 and therefore

$$
0=0^{\top} x=\left(1^{\top} M\right) x=1^{\top}(M x),
$$

where $1^{\top} M x$ is the sum of the coordinates of $M x$, all nonnegative by the condition, and therefore there is equality throughout. Thus $M x=0, x \geq 0$, that is, $x$ is a circulation and it is an integer vector: there exists a multiset $\mathcal{C}$ of circuits with $x=\sum_{C \in \mathcal{C}} C$. Moreover, because of $M^{+} x \geq 1$, every element is covered by at least one of the circuits.

Conversely, for any multiset $\mathcal{C}$ of circuits that cover the vertex-set $x:=\sum_{C \in \mathcal{C}} C$ is an integer vector that satisfies $(\mathrm{P})$. Moreover,

$$
c^{\top} x=\sum_{C \in \mathcal{C}} c(C)=\sum_{C \in \mathcal{C}} \operatorname{ind}(C),
$$

establishing the claim.

Claim 2: The dual optimum of $(\mathrm{P})$ is equal to the right hand side of the minmax equality of the theorem.

An integer dual solution is of the form $(\pi, y), \pi \in \mathbb{Z}^{n}, y \in \mathbb{Z}^{n}$, where $\pi$ is a potential for $c(a)-y_{h(a)}$ (see the definition of potentials). Such an integer potential exists for an integer $y$ if and only if there is no negative circuit for the edge-weights $c(a)-y_{h(a)}(a \in A)$, that is, if and only if for every circuit $C$,

$$
\sum_{v \in V(C)} y_{v}=\sum_{a \in C} y_{h(a)} \leq \sum_{a \in C} c(a)=\operatorname{ind}(C)
$$

that is, if and only if $y$ is an index-bounded weighting. The dual objective value is $\sum_{v \in V} y_{v}$. 


\section{Cyclic Colourings}

The authors of [1] prove a theorem that can be considered to be the "antiblocker" of Theorem 3.3, and actually a sharpening of such a theorem. We provide a simple proof of this theorem as well, in the spirit of the the proof of Theorem 3.3, and using Theorem 2.2. (Our definitions and terminology are slightly different from [1]: the definitions do not depend on the orientation, so they concern only undirected graphs; the terminology and notation are simplified and unified.)

Let $G=(V, E)$ be an undirected graph. According to Zhu [6] a circular $r$-coloration of $G$ is a function $f: V \longrightarrow[0, r)=(\{x \in \mathbb{R}: 0 \leq x<r\})$, such that for all $(x, y) \in A$ : $\operatorname{dist}(x, y) \geq 1$, where $\operatorname{dist}(x, y):=\operatorname{dist}_{f, r}(x, y):=\min \{|f(x)-f(y)|, r-|f(x)-f(y)|\}-$ the distance of $(x, y)$ on the circle of perimeter $r$.

If a cyclic order $O$ is given, then a circular $r$-coloration is called a cyclic $r$-coloration (with respect to the cyclic order $O$ ) if in addition the order $\left(v_{1}, v_{2}, \ldots, v_{n}\right), 0 \leq f\left(v_{1}\right) \leq f\left(v_{2}\right) \leq \ldots \leq f\left(v_{n}\right)<r$ is equivalent to $O$. We will use these terms for directed graphs as well, whenever the properties hold for the underlying undirected graph.

The infimum (which is clearly a minimum) of all reals $r>0$ such that $O$ has a circular $r$-colouring is the circular chromatic number, denoted by $\chi_{\text {circ }}$. The minimum of $r>0$ for which there exists a cyclic $r$-coloring (with respect to $O$ ) will be denoted by $\xi_{O}$. (It is easy to check that $\chi_{\text {circ }} \leq \chi \leq\left\lceil\chi_{\text {circ }}\right\rceil \leq\left\lceil\xi_{O}\right\rceil$, where $\chi=\chi(D)$ is the (usual) chromatic number.)

We define for any circuit $C$, the cyclic length of $C$ as $l_{O}(C):=|C| / \operatorname{ind}(C)$ (with respect to the fixed cyclic order $O$ ).

Theorem 4.1 Let $D$ be a nontrivial strongly connected digraph and $O$ a coherent cyclic order on its vertices. Then

$$
\xi_{O}(D)=\max \left\{l_{O}(C): C \text { a circuit of } D\right\}
$$

Proof: Let $D=(V, A)$ be a digraph, with vertex set $V$, and let $\left(v_{1}, \ldots, v_{n}\right)$ be a linear order of $V$, which is an opening of the coherent cyclic order $O$. We denote the $\operatorname{arc}$ set of $D$ by $A=\left\{a_{1}, \ldots, a_{m}\right\}$.

Consider the linear program $x \in \mathbb{R}^{m}$, maximize $\sum_{i=1}^{m} x_{i}$ subject to $M x \leq 0, x(B) \leq 1, x \geq 0$

where $B \subseteq A$ is the set of backward arcs. Clearly, this linear program is feasible and bounded.

Claim 1: The primal optimum of $\left(\mathrm{P}^{*}\right)$ is equal to the right hand side of the theorem.

Indeed, again, $M x \leq 0$ implies $M x=0$, so primal solutions are circulations $x$ with $x(B) \leq 1$. Since $D$ contains at least one circuit, and for any circuit $C, C / \operatorname{ind}(C)$ is a primal solution (vertex) of $(\mathrm{P})$ with objective value $|C| / \operatorname{ind}(C)=l_{O}(C)$, the primal optimum is positive, and greater than or equal to the right hand side of the theorem.

Conversely, any primal solution $x$ is a nonnegative circulation, that is, a nonnegative linear combination of circuits. We write $x=\sum_{C \in \mathcal{C}} \lambda_{C} C,\left(\lambda_{C} \geq 0\right)$, for some set $\mathcal{C}$ of circuits. The constraint $x(B) \leq 1$ is equivalent to $\sum_{C \in \mathcal{C}} \lambda_{C} \operatorname{ind}(C) \leq 1$ and therefore :

$$
1^{\top} x=\sum_{C \in \mathcal{C}} \lambda(C)|C|=\sum_{C \in \mathcal{C}} \lambda(C) \operatorname{ind}(C) l_{O}(C) \leq \max \left\{l_{O}(C): C \text { a circuit of } D\right\}
$$

finishing the proof of the claim. 
Note that Claim 1 does not use that the given order is coherent. This will be exploited for Claim 2.

Fix $\left(\pi_{1}, \ldots, \pi_{n}, r\right)$ to be the dual optimum. Starting with this vector we construct a cyclic colouring.

According to Claim 1, $r=\max \left\{l_{O}(C): C\right.$ a circuit of $\left.D\right\}>1$.

Claim 2: For every forward arc $u v, \quad 1 \leq \pi_{v}-\pi_{u} \leq r-1$.

For every backward arc $u v, \quad 1 \leq \pi_{u}-\pi_{v} \leq r-1$.

First, $\left(\pi_{1}, \ldots, \pi_{n}, r\right)$ satisfies the dual constraints for each $a=u v \in A$, that is :

$$
\pi_{v}-\pi_{u} \geq \begin{cases}1 & \text { if } u v \text { is a foward arc } \\ 1-r & \text { if } u v \text { is a back arc }\end{cases}
$$

Furthermore, if $u v$ is a backward arc, by coherence, there exists a forward path $P$ between $v$ and $u$, and adding up the inequalities concerning the arcs of this path: $\pi_{u}-\pi_{v} \geq|P|-1 \geq 1$.

Likewise, if $u v$ is a forward arc, $u v$ lies in a circuit $C$ on index 1. Let $u^{\prime} v^{\prime}$ be the unique backward arc of $C$. Then $\pi_{v^{\prime}} \leq \pi_{u} \leq \pi_{v} \leq \pi_{u^{\prime}}$, and therefore $\left|\pi_{u}-\pi_{v}\right| \leq\left|\pi_{u^{\prime}}-\pi_{v^{\prime}}\right| \leq r-1$. This finishes the proof of Claim 2.

For any dual solution $\left(\pi_{1}, \ldots, \pi_{n}, r\right)$ of $(\mathrm{P})$ define $q: V(D) \longrightarrow[0, r)$ with $\pi_{i}=: p\left(v_{i}\right) r+q\left(v_{i}\right)$, that is, $q\left(v_{i}\right)$ is the remainder of $\pi_{i}$ modulo $r$. It is straightforward to check that Claim 2 implies that $q$ is a circular $r$-coloration. Moreover, the linear order $O_{\pi}$ of the vertices defined by the increasing order of $\pi_{v},(v \in V)$ has the same set of backward arcs as $O$, so these two orders are equivalent (by Theorem 2.2.)

Claim 3: The function $q$ is a cyclic $r$-coloration with respect to $O$.

In addition to Claim 2 we have to check that $q$ defines a cyclic order equivalent to $O$. According to Theorem 2.1, it is sufficient to check that in the linear order $O_{q}$ where the vertices are in increasing order of $q$, every circuit has exactly the same number of backward arcs as in $O$, that is, as in $O_{\pi}$, since the latter two have been proved to be equivalent. Let $C$ be an arbitrary circuit. Thanks to Claim 2, we know that for an arc $u v, p(u)-p(v)$ equals either $-1,0$, or 1 . Clearly, $\operatorname{arcs} u v$ with $p(u)=p(v)$ are forward arcs or backward arcs in both $O_{\pi}$ and $O_{q}$; we also see from Claim 2 that in case $p(u)-p(v)=1, u v$ is a backward arc in $O_{\pi}$, and it is a forward arc in $O_{q}$; similarly, if $p(u)-p(v)=-1$, then $u v$ is a forward arc in $O_{\pi}$, and a backward arc in $O_{q}$; since $\sum_{u v \in C} p(u)-p(v)=0$, we have :

$$
|\{u v \in C: p(u)-p(v)=1\}|=|\{u v \in C: p(u)-p(v)=-1\}|,
$$

that is, the number of backward arcs remains the same in $O_{q}$ and $O_{\pi}$ in every circuit.

Claims 1 and 3 assert that $\max \left\{l_{O}(C): C\right.$ a circuit of $\left.D\right\}=r \geq \xi_{O}(D)$. To finish the proof of the theorem note first that for any cyclic $\xi_{O}(D)$-coloration, and any circuit $C$, with the distances defined by the cyclic coloration, $|C| \leq \sum_{x y \in A(C)} \operatorname{dist}(x, y)=\xi_{O}(D) \operatorname{ind}(C)$.

With the starting idea of defining a coloring from a potential, the proof can be finished in two ways, see [2] and [5]. The former checks directly that the order defined above is cyclically equivalent to the given one. The latter proves that with a suitable choice of $x_{0}$, the distances from $x_{0}$ are all in the interval $[0, r)$. We have chosen here a third, simpler way. 
Acknowledgment: The authors wish to thank Adrian Bondy for suggesting a purely linear programming approach to Theorem 3.3 - a suggestion that was independent from the network flow approach in [5], led to a different proof, and was a crucial motivation for the present work.

\section{References}

[1] S. Bessy and S. Thomassé, Spanning a strong digraph by $\alpha$ circuits: A proof of Gallai's conjecture, Combinatorica, to appear.

[2] P. Charbit, $\mathrm{PhD}$ thesis, Lyon University, in preparation.

[3] T. Gallai, Problem 15, in Theory of Graphs and its Applications (M. Fiedler, ed.), Czech. Acad. Sci. Publ., 1964, p. 161.

[4] A. Schrijver, Combinatorial Optimization, Springer, 2003.

[5] A. Sebő, Minmax Relations for Cyclically Ordered Graphs, Cahiers du Laboratoire Leibniz, Septembre 2004.

[6] X. Zhu, Circular chromatic number: a survey, Discrete Mathematics, 229 (1-3), (2001), pp. 371-410. 


\section{Les Cahiers Leibniz}

Le Laboratoire Leibniz est fortement pluridisciplinaire. Son activité scientifique couvre un large domaine qui comprend des thèmes fondamentaux aussi bien en informatique qu'en mathématiques, avec une ouverture sur l'apprentissage machine, la modélisation de systèmes complexes adaptatifs, et les applications aux environnements informatiques pour l'apprentissage humain.

Les Cahiers Leibniz ont pour vocation la diffusion de rapports de recherche, de supports de cours, de textes de séminaires ou de projets de publications réalisés par des membres du laboratoire. Ils peuvent accueillir aussi des textes de chercheurs n'appartenant pas au laboratoire Leibniz mais qui travaillent sur des thèmes proches et ne disposent pas de tels supports de publication. Ces chercheurs sont priés de contacter un des membres du comité éditorial; le comité décidera de l'acceptation du texte proposé.

Le contenu des textes publiés dans les Cahiers Leibniz relève de la seule responsabilité de leurs auteurs.

The research at Laboratoire Leibniz is multidisciplinary. It covers a large domain of fundamental and applied subjects in informatics an mathematics, with openings to machine learning, the modelisation of adaptive complex systems, and applications to teaching software.

The Cahiers Leibniz aim at diffusing research reports, lectures, and texts of conferences or pre-prints of the members of the laboratory. Moreover, the Cahiers welcome manuscripts of researchers belonging to other laboratories, working on subjects close to ours, but not disposing of such a medium. These researchers should contact one of the members of the editorial board; the latter will decide whether to accept the proposal.

The responsibility of the contents of the Cahiers lies exclusively with the authors.

\section{Comité éditorial}

Mirta B. Gordon (responsable), Annie Bessot, Gerd Finke, Humbert Fiorino, Denise Grenier, Philippe Jorrand, Andras Sëbo

\section{Directeur de la publication}

Nicolas Balacheff 
